\title{
BMG Global Health Addressing the challenges of diagnostics demand and supply: insights from an online global health discussion platform
}

\author{
Nora Engel, ${ }^{1}$ Keri Wachter, ${ }^{2}$ Madhukar Pai, ${ }^{3}$ Jim Gallarda, ${ }^{4}$ Catharina Boehme, ${ }^{5}$ \\ Isabelle Celentano, ${ }^{6}$ Rebecca Weintraub ${ }^{2,6}$
}

To cite: Engel N, Wachter K, Pai $\mathrm{M}$, et al. Addressing the challenges of diagnostics demand and supply: insights from an online global health discussion platform. BMJ Global Health 2016;1: e000132. doi:10.1136/ bmjgh-2016-000132

Received 19 July 2016 Revised 1 November 2016 Accepted 3 November 2016

\section{CrossMark}

${ }^{1}$ Department of Health, Ethics \& Society/CAPHRI,

Maastricht University,

Maastricht, The Netherlands

${ }^{2}$ Harvard Medical School,

Global Health Delivery Project at Harvard University, Boston, Massachusetts, USA

${ }^{3}$ McGill Global Health Programs \& McGill International TB Centre, McGill University, Montreal, Canada

${ }^{4}$ Bill and Melinda Gates Foundation, Seattle, Washington, USA

${ }^{5}$ Foundation for Innovative New Diagnostics, Geneva, Switzerland

${ }^{6}$ Brigham and Women's

Hospital, Boston

Massachusetts, USA

\section{Correspondence to}

Dr Nora Engel;

n.engel@maastrichtuniversity. $\mathrm{nl}$

\section{ABSTRACT}

Several barriers challenge development, adoption and scale-up of diagnostics in low and middle income countries. An innovative global health discussion platform allows capturing insights from the global health community on factors driving demand and supply for diagnostics. We conducted a qualitative content analysis of the online discussion 'Advancing Care Delivery: Driving Demand and Supply of Diagnostics' organised by the Global Health Delivery Project (GHD) (http://www.ghdonline.org/) at Harvard University. The discussion, driven by 12 expert panellists, explored what must be done to develop delivery systems, business models, new technologies, interoperability standards, and governance mechanisms to ensure that patients receive the right diagnostic at the right time. The GHD Online (GHDonline) platform reaches over 19000 members from 185 countries. Participants $(\mathrm{N}=99)$ in the diagnostics discussion included academics, non-governmental organisations, manufacturers, policymakers, and physicians. Data was coded and overarching categories analysed using qualitative data analysis software. Participants considered technical characteristics of diagnostics as smaller barriers to effective use of diagnostics compared with operational and health system challenges, such as logistics, poor fit with user needs, cost, workforce, infrastructure, access, weak regulation and political commitment. Suggested solutions included: health system strengthening with patientcentred delivery; strengthened innovation processes; improved knowledge base; harmonised guidelines and evaluation; supply chain innovations; and mechanisms for ensuring quality and capacity. Engaging and connecting different actors involved with diagnostic development and use is paramount for improving diagnostics. While the discussion participants were not representative of all actors involved, the platform enabled a discussion between globally acknowledged experts and physicians working in different countries.

\section{INTRODUCTION}

There is broad consensus among patients and providers that health systems need

\section{Key questions}

What is already known about this topic?

- The potential of diagnostics in low and middle income countries is not fully realised and diagnostics are not necessarily available, accessible or affordable to those in need.

What are the new findings?

- The participants of an innovative global health discussion platform discussed a universal list of challenges along the value chain of diagnostics in low and middle income countries, complemented with an innovative set of solutions.

\section{Recommendations for policy}

- Ensuring broader access to diagnostics requires a seamless supply chain and a strengthened health system with absorption capacity for diagnostic innovation.

- Innovation processes need to also include end-users and service innovations, the local evidence base needs to be expanded, and guidelines and evaluation processes need to be harmonised.

broader access to appropriate diagnostic tools. Globally, commercial entities have developed diagnostics to identify the presence and cause of disease and determine a treatment course. Investment in diagnostic technologies, largely concentrated in the US and European markets, has increased the supply and sophistication of diagnostic technologies, including the ability to differentiate between diseases that present with similar symptoms, identify the presence of latent infectious agents, and provide practical low-cost monitoring for treatment efficacy and disease recurrence. In addition, new diagnostics have identified response rates to vaccinations and treatment-resistance patterns. While diagnostics have become key 
drivers to generate value for patients and health systems, this potential has not been met across all settings, particularly in low and middle income countries (LMICs).

Many have observed that diagnostics development has stalled for several neglected diseases, and even where good tests are available, they are not necessarily accessible or affordable to those who need them the most. For example, of the 9.6 million tuberculosis (TB) cases each year, 3 million are either not diagnosed or not notified. ${ }^{1}$ Only one-third of the estimated patients with multidrug-resistant TB are diagnosed. Only about half of those infected with HIV are aware of their status. ${ }^{2}$ In half of malaria-endemic African countries, over $80 \%$ of malaria treatments are applied without diagnostic testing. ${ }^{3}$ This paper reports on a discussion on the bottlenecks and opportunities in global diagnostics on Global Health Delivery Online (GHDonline) (http:// www.ghdonline.org/), a professional virtual community founded in 2008 by the Global Health Delivery Project (GHD) at Harvard University (http://www. globalhealthdelivery.org/), aimed at enabling researchers and practitioners to share data, expertise and resources widely and quickly. Professional virtual communities-online professional networks first used in the business world-create important opportunities for knowledge exchange between professionals from disparate settings and across multiple organisations, industries, and geographies. ${ }^{4}$ They are a type of 'community of practice $^{5}$ that can help identify new research questions and promote testing of new ideas and tools. Over time, virtual communities become more cohesive, and members can leverage them to advocate for emerging industry standards.

The GHDonline professional virtual communities address common challenges in healthcare delivery-how to integrate new treatment protocols, employ quality improvement measures, incorporate emerging research, or address clinical challenges or infrastructural needs, among others. Today, GHDonline hosts more than 19000 professionals from 185 countries representing more than 5200 organisations. Members include physicians, researchers, nurses, programme officers, engineers, policymakers, architects, librarians, economists, and others representing an array of experiences. Expert Panels on GHDonline are virtual, week or 2-week-long events that bring together leading experts to share their knowledge and discuss key issues in the field. These events are free, open to the public, accessible anywhere, asynchronous, and allow for dialogue between leading experts and community participants. The GHDonline Expert Panel discussion, 'Advancing Care Delivery: Driving Demand and Supply of Diagnostics,' was organised to discuss approaches to ensure that novel diagnostics reach the populations they are designed for. The discussion explored what must be done to develop delivery systems, business models, new technologies, interoperability standards, and governance mechanisms to ensure each patient will receive the right diagnostic at the right time. In this paper we present a thematic analysis of the discussion content (box 1 for more details on the discussion and data analysis).

We grouped the discussions into major themes of addressing challenges of diagnostics demand and supply. Figure 1 provides a summary of the main challenges in the diagnostics value chain in LMICs that were discussed. Figure 2 provides a summary of the key potential solutions suggested by the Expert Panel discussion members. Below we discuss these challenges and solutions in an integrated manner. Participants did not assign solutions to specific actors along the diagnostic value chain. However, theme 1-5 could be addressed by donors, regulators, guideline and decision makers, nongovernmental organisations (NGOs) and academics. Manufacturers and donors who support them are most suited to improve inclusion of end-users into design processes. Manufacturers and government officials (health, finance, industry) are well-positioned to tackle the market shortcomings and supply chain innovations required to ensure reliable manufacturing and supply (theme 4 and 5); while governments could tackle the solutions listed under quality (theme 6).

\section{POINT-OF-CARE (POC) TESTING NEEDS A FUNCTIONING DIAGNOSTIC/HEALTH SYSTEM}

Diagnostics need strong systems to run on even if they are used at POC (ie, near patients). Otherwise there is limited impact on patient outcomes. The challenges that health systems must address to ensure access to effective POC testing are myriad and complex. In the discussions on different countries' approaches to implementing diagnostics, participants mentioned challenges to effective use of tests such as cost, staff capacities, irregular supply, transportation delays, inconsistent funding by governments and donors, poor forecasting and stock management, quality assurance, as well as problems with infrastructure, access and end-user utility. Technical aspects of diagnostics such as low accuracy, throughput, turn-around-time, or poor quality were far less mentioned in these discussions. Participants also recognised that some POC tests have put additional stress on the already weak health systems (\#211). The health system is challenged even more if POC testing requires instruments (calibration, maintenance, etc), as opposed to instrument-free rapid diagnostic tests (RDTs) such as lateral flow assays. Yet, simply having an RDT does not ensure linkages to care or acting on the result. One participant warned against viewing POC testing as a panacea. Rather than attempting to support the full range of clinical needs with POC testing, it should be used to complement a functioning diagnostic system (\#18). Furthermore, the nature of the existing diagnostic ecosystem (centralised vs testing in smaller, peripheral laboratories) matters for the challenges that POC testing faces (\#209). 89

Participants thus emphasised strengthening the ( primary) health system to improve delivery and use of 


\section{Box 1 Characteristics of the online discussion and data analysis}

Global Health Delivery Online (GHDonline) hosted a 2-week Expert Panel focusing on diagnostic solutions for global health (archived and available at http://www.ghdonline.org/global-diagnostics/discussion/driving-demand-and-supply-of-diagnostics/). This site-wide discussion involved 12 expert panellists (see Acknowledgements section) that were selected in advance to prime and moderate the discussion. It was open to the entire GHDonline network-16509 GHDonline members at the time-60\% of which work or reside outside of the USA. The event received more than 2300 unique visitors during the 2-week time frame, and 99 individuals provided 219 written comments and shared 25 resources during the discussion. These 99 participants who contributed directly to the conversation with written comments, work across the globe-33 in the USA, 38 in Africa, 13 in Asia, 8 in Latin America/the Caribbean, 10 in Europe, and 2 in Australia. Of these participants, 14 were affiliated with hospitals or clinics, 19 with Ministries of Health or governmental organisations, 30 with universities or academic centres, 15 with research institutions, 19 with non-governmental organisations or non-profits, 1 with a donor group, 7 with industry, and 6 participants identified as freelancers unaffiliated with an organisation (participants could name multiple locations and/or affiliations). The intent was to explore methods for driving innovation and access in global health diagnostics, with special emphasis on meeting the needs of the decision-making demand stakeholders (ie, countries) with technology solutions that could be used at lower levels of the healthcare system (ie, point-of-care testing). The areas of exploration in this regard included data technologies/platforms, business models, interoperability standards, as well as other enabling regulatory or governance mechanisms.

The questions that guided the discussion (see below) were derived from the diagnostic value chain by Derda et af which covers demand and initial assessment, research and development, evaluation and adaptation, regulation via national and international bodies and product, quality assurance/control and delivery support. The discussion questions did not focus on individual diseases specifically, but gave tuberculosis (TB), malaria, HIV and human papillomavirus (HPV) as key examples, and asked for challenges in the general diagnostic set-up of countries. The entire online discussion was analysed by qualitative content analysis which allows identifying overarching themes across the different responses and discussion points. ${ }^{7}$ A coding list was developed based on the discussion questions, the framework guiding the questions ${ }^{6}$ and the codes that emerged on reading the material. Data were coded by the first author using qualitative data analysis software NVivo and further analysed by grouping codes under higher order headings and categories ${ }^{7}$ described in the discussion section. The analysis was further refined and cross-checked in consultation with the coauthors to enhance inter-rater reliability. Data sources are indicated by the number of the online entry on the discussion platform.

The following discussion questions were posed to the GHDOnline community during the 2-week period:

1. What is your country's approach for using diagnostics for HIV, TB, malaria, and HPV? Please explain for each of the following

- Decentralised point-of-care testing?

Decentralised sample collection followed by reference laboratory centralised testing?

- Predominant empiric treatment?

2. What do you see as the biggest barriers to effective use of diagnostics? What would it take to remove these barriers and expand access?

3. How have diagnostics for HIV, TB, malaria, and HPV been validated in the country where you work? Was operational or implementation research conducted for these diagnostics? How was it done and what lessons were learnt?

4. Do you see advantages to importing, versus locally manufacturing, diagnostics?

5. How are new diagnostics introduced in your country, and who makes decisions about which diagnostics will be covered by insurance schemes (if applicable)?

6. How are guidelines for use disseminated? What challenges have you seen in adapting global guidelines to your setting, and what strategies have you found for training providers on these guidelines?

7. What policies regulate or impact the use of diagnostics in your setting?

8. What lessons would you offer regarding production supply, quality, service and support, or other post-launch needs for diagnostics?

9. To ensure the right patient gets the right diagnostic test and appropriate treatment, what would the ideal delivery system look like for HIV,

$\mathrm{TB}$, malaria and HPV in your setting?

diagnostics as most important (for instance \#197), including strengthening infrastructure and regulatory systems (\#199). This will also enhance quality and diagnostic confidence among end-users (\#51). Health system strengthening as propagated by the WHO focuses on specific building blocks (see the WHO framework, http://www.who.int/healthsystems/strategy/everybodys_ business.pdf), but an understanding of how aspects such as human resources, available technologies, finances, governance, routines and infrastructure interact is lacking from these building blocks. These dynamics come to the fore when examining diagnostic and care processes (\#209).

A patient-centred and integrated approach to service delivery should guide health system strengthening efforts (\#61). Such an approach would recognise that disease management is not purely medical but also involves social, psychological, behavioural, and financial aspects. Consequently, diagnostics can only be one part of that solution, necessary but not sufficient (\#131). ${ }^{10} \mathrm{~A}$ patient-centric solution requires analysis of the entire value chain of care delivered, an understanding of the roles that diagnostics, treatment, counselling, community outreach, etc, play, how to allocate resources or incentives accordingly, and how to ensure professionals work closely together in an integrated system rather than in professional silos $(\# 214,215,131)$.

The capacity needed to create functioning diagnostic services runs somewhat contrary to what most POC tests promise, namely to work where there is insufficient laboratory infrastructure, limited human resources, and prolonged turn-around on laboratory-based test results. 
Point of care diagnostic test value chain

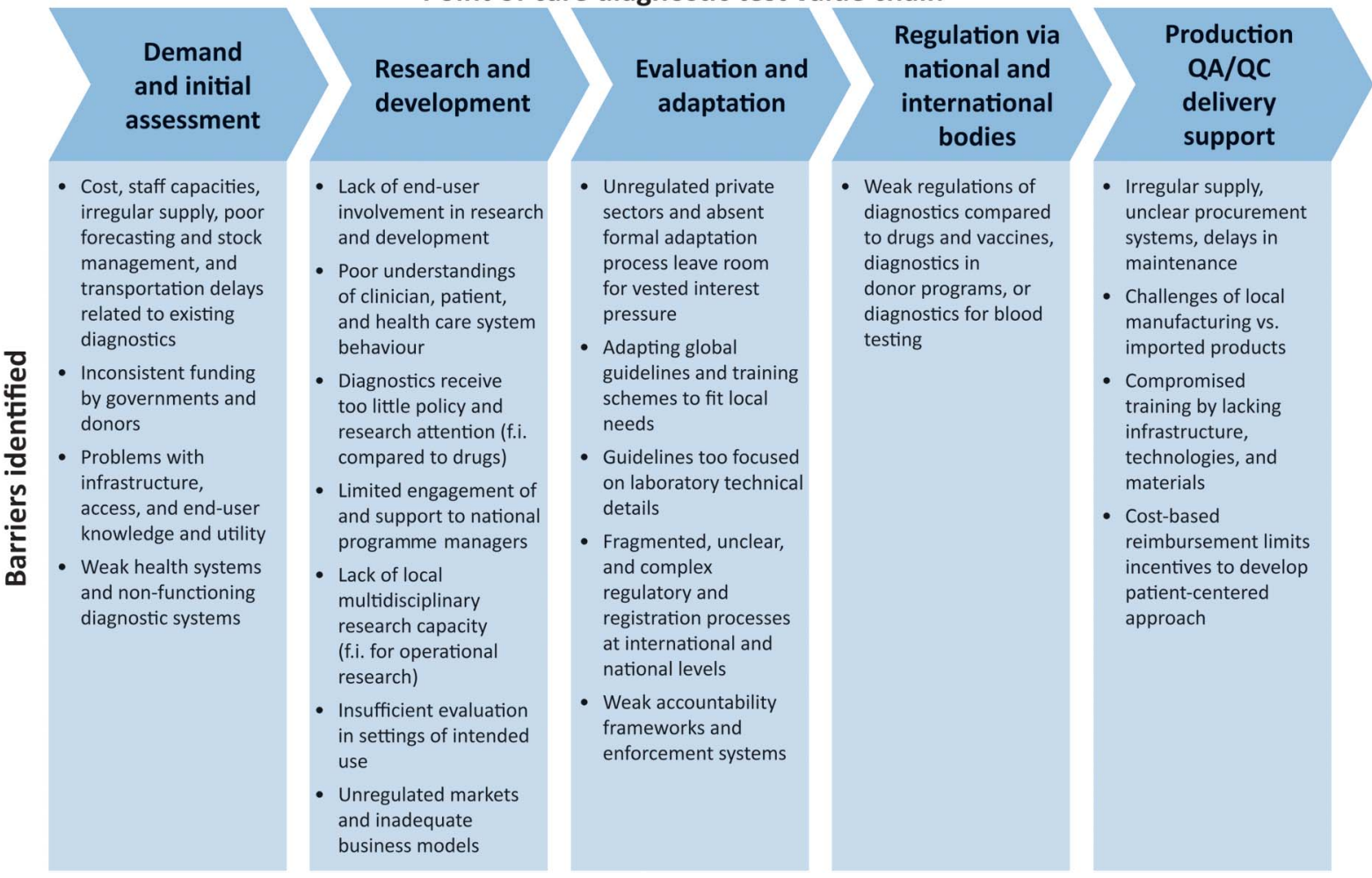

Figure 1 Barriers along the diagnostics value chain, based on the GHDonline Expert Panel discussion analysis. GHDonline, Global Health Delivery Online; QA/QC, quality assurance/quality control.

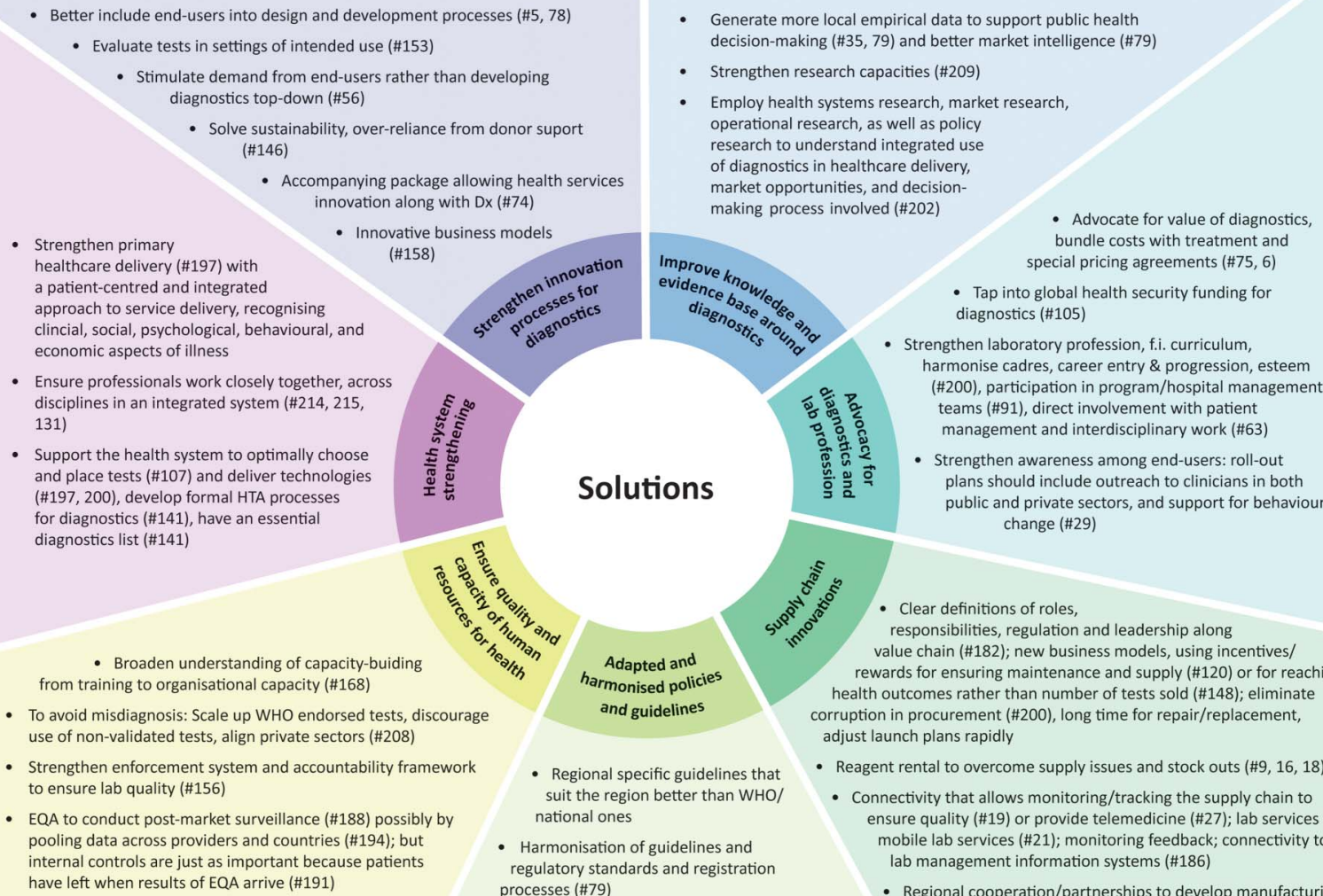

- Support the health system to optimally choose and place tests (\#107) and deliver technologie $(\# 197,200)$, develop formal HTA processes for diagnostics (\#141), have an essential diagnostics list (\#141)

- Strengthen laboratory profession, fi.i. curriculum harmonise cadres, career entry \& progression, esteem (\#200), participation in program/hospital management teams (\#91), direct involvement with patient management and interdisciplinary work (\#63)

- Strengthen awareness among end-users: roll-out plans should include outreach to clinicians in both public and private sectors, and support for behaviour change $(\# 29)$

Solutions from training to or

- To avoid misdiagnosis: Scale up WHO endorsed tests, discourage use of non-validated tests, align private sectors (\#208)

- Strengthen enforcement system and accountability framework to ensure lab quality (\#156)

- EQA to conduct post-market surveillance (\#188) possibly by pooling data across providers and countries (\#194); but internal controls are just as important because patients have left when results of EQA arrive (\#191)

Figure 2 Solutions that were suggested by GHDonline Expert Panel participants. EQA, external quality assessment; GHDonline, Global Health Delivery Online; HTA, health technology assessment. 
Despite their promise of simplicity, rapidity and low cost, POC devices are not enough to overcome absent or nonfunctioning health system capacity for diagnosing. Instead, if POC testing is carried out at new testing sites, those sites need to be linked to existing laboratories and embedded into existing supply, distribution and service systems. These are to some extent the diagnostic set-ups and practices that POC diagnostics were meant to sidestep (\#209).

\section{STRENGTHENING INNOVATION FOR DIAGNOSTICS}

Include end-users in design processes: Ensuring end-users (mainly discussed as clinicians) are aware of tests and able to use them, overcoming widespread empirical treatment, and ensuring test results are linked to care, were priorities highlighted by many participants. Low demand of new diagnostics by end-users is, according to some participants, caused by a lack of knowledge of policy changes (\#29, 76) and the importance and use of new tests (\#94). In contrast, others emphasised that physicians and patients need to have trust in the test results and the quality of laboratory work. Diagnostic confidence can be low in settings with inadequately funded laboratory infrastructure, short testing menus and reputations of questionable accuracy (\#51). Participants agreed that end-user needs, beliefs and behaviours matter, and that end-users should be included in the design process (\#5, 78). However, currently diagnostics are mainly provided in a top-down manner, 'from tech companies and their agents, to government agencies or hospitals, to laboratories, then clinicians, and then patients,' instead of stimulating demand for diagnostics from patients and providers (\#56). Test developers often do not know much about the settings they are developing tests for and only recently have some developers begun testing their prototypes in low-resource settings (\#157), while market opportunities remain poorly defined $(\# 79,5)$. Several participants cited the example of Xpert MTB/RIF, a diagnostic test for TB that is often deployed in settings that do not match its intended use. It requires electricity, air conditioning, consumables with short shelf life and remote management of the machine (\#5) and is thus not fit-for-purpose at POC. It was suggested that health systems research, market research, operational research as well as policy research might help in understanding the integrated use of diagnostics in healthcare delivery, market opportunities and decision-makings processes involved (\#202).

Advocate for an increased focus on diagnostics in research and policy: Several participants pointed out that diagnostics do not receive the same policy and research attention as drug development, and if they do, attention is mainly centred on HIV, TB and malaria. The latter are mostly organised in vertically managed disease control programmes with separate funding streams, diagnostic laboratories and testing facilities (\#38). This lack of attention to diagnostics was identified both in the global health policy world and within clinics and hospitals. Several participants mentioned how physicians should advocate for having tests available where they work (\#6), how laboratory professionals should be a part of programme management teams (\#91), how the diagnostic community should emphasise the cost-saving potential of testing to public health (\#96) and how laboratory workers or radiologists should interact more with patients directly (\#63). Furthermore, the laboratory profession needs to be strengthened and made more attractive to potential workers (\#63).

\section{IMPROVING KNOWLEDGE BASE AND EVALUATION PROCESSES}

Adapting delivery systems and POC testing programmes to a particular country context requires research capacities, platforms where insights can be shared, and funding (\#209). Participants agreed that operations research and health system research are becoming more important as new diagnostic tests are more widely available. National TB programmes, for instance, increasingly require locally generated data to adopt and scale up new diagnostics despite globally available evidence and guidelines (\#158). However, the combined technical and operational evaluations of tests require multidisciplinary teams of researchers which are difficult and expensive to assemble in contexts of lacking capacities (\#102, 109), and protocol approval processes can be time-consuming (\#111). Furthermore, POC tests should be evaluated in their setting of intended use and not in a laboratory (\# 153,) or in a country with very different resources (\#157).

In many low-resource settings, regulation and financial models for diagnostics are weak. In Nigeria for instance, the Ministry of Health supplies primary health facilities with diagnostics when the clinic is being built, but maintenance is done by donors and NGOs and thus potentially less sustainable (\#4). Public sectors often do not have a formal process of introducing diagnostics, leaving room for vested interest pressures from outside donors, interest groups or manufacturers (\#141). This concern was echoed by other participants as well. Some participants mentioned that tests within donor/NGO-controlled programmes are strictly regulated and that use of diagnostics is influenced by endorsements from institutions such as WHO or Centres for Disease Control and Prevention (CDC) (\#180). Most countries have stronger regulations for vaccines, drugs, biologicals, and diagnostics related to blood safety testing (\#176). This should be seen as an opportunity for better regulation of diagnostics (\#180). Alleviating weak regulation would require harmonisation of regulatory and registration processes, possibly in regional attempts. Formal, transparent health technology assessment processes with stewardship and governance by the Ministry of Health, locally generated evidence informing decisions and buy-in from all the stakeholders has helped in the past (\#108). 


\section{ADDRESSING MARKET SHORTCOMINGS}

Many participants highlighted cost as a major barrier to conducting tests, maintaining equipment and affording consumables. However, driving costs so low that manufacturers leave the market, as is happening with malaria RDT kits, is not a solution. ${ }^{11}$ Thus, especially in the public sector, sustained advocacy around the value of diagnostics is needed, for instance by advocating for diagnostics and treatment together (ie, test and treat), and to increase budgets of testing programmes. To better justify these investments, some participants suggested that cost considerations for diagnostics should include costs saved by the health system, for example as a result of decreased treatment cost (\#28), and consider reimbursement of diagnostics based on, for instance, the clinical value they provide (\#203, \#101). This would also incentivise development of new tests (\#203). Increased collaboration between clinical personnel and health economists would make cost-effectiveness analyses stronger and more credible (\#106). In the private sector, special pricing agreements initiatives (eg, the Initiative for Promoting Affordable and Quality TB Tests (IPAQT) (http://www.ipaqt.org) model in India) need to be established to make tests more accessible (\#75). In general, different challenges compound one another. One discussant pointed out how the inadequate funding of laboratory services is related to the underrepresentation of laboratory professions in hospital decision-making processes (\#90).

\section{SUPPLY CHAIN INNOVATIONS}

Participants argued for clear definitions of roles, responsibility, regulation and leadership along the value chain (\#182) and clear procurement systems (\#186). In order to overcome supply issues and stock outs, the concept of reagent rentals (ie, adding a surcharge to every use of test reagents to cover instrument capital expenses) seemed to draw support from members in Mexico, East Africa, and Asia (\#91618). Others voiced concerns about the possible monopoly practice character of reagent rental (\#151). Connectivity and related telemedicine solutions were mentioned to monitor or link mobile laboratory services to centralised laboratories until health systems are strengthened (\#19, 21, 27) or to use smart tracking devices to monitor quality of supplies (\#19). One participant involved with telemedicine solutions voiced concerns about the challenges of reading laboratory images online (\#31).

Participants also discussed the advantages and disadvantages of local versus centralised manufacturing. The idea of regional partnerships to develop regional manufacturing capacity $(\# 119,152)$ was appreciated among participants (\#134), for instance, within the East African Community (\#139). Yet, participants also discussed that having a mix of imported and regionally/locally manufactured products might make more sense and be more cost-effective (\#118), especially for middle income countries and emerging economies (\#122). Ensuring local supply possibly through local business models (for instance incentives, social impact bonds or social health insurance schemes tied to health outcomes rather than number of tests sold) could be more important than local manufacturing (\#120, 148). Others warned that this would require eliminating corruption in procurement first (\#200).

\section{ENSURING QUALITY AND BUILDING CAPACITY IN DIAGNOSTICS IMPLEMENTATION}

Participants emphasised that a broader understanding of capacity-building is required. This involves training laboratory technicians, and ensuring all healthcare workers along the diagnostic process understand their roles and responsibilities (\#174) and building organisational capacity (\#168). To overcome lack of staff capacities, manufacturers were called to improve (\#163) and invest in training of the workforce on their instruments (\#63). It was emphasised that global guidelines and training schemes need to be adapted to fit national and local settings (\#154), because they tend to address technical laboratory details and intended use in general terms. However, they do not, for instance, address how different healthcare workers can request a test (\#174) or provide sufficient support for scale-up (\#158). While participants agreed that poor training undermines quality of diagnosing (\#163), strengthening enforcement systems and accountability frameworks in private and public sectors to ensure diagnostic quality may be more important (\#156). Both national and international efforts to regulate the quality of diagnostics need to be strengthened to prevent low-quality products from being sold (\#211) and for laboratories to be well managed (\#192). WHO-endorsed tests need to be scaled up and use of non-validated tests should be discouraged in public and private sectors (\#208).

\section{CONCLUSION}

The panel identified challenges and solutions along the global health diagnostics value chain. The challenges discussed largely overlap with what has been described in the literature on diagnostic test development for TB and HIV. ${ }^{12-15}$ The discussion confirmed that these challenges indeed are universal and similar across diseases and countries, despite different national institutions and processes (for regulation, quality control, etc). These challenges provide to some extent answers as to why new tests such as Xpert MTB/RIF for TB are not as widely and successfully implemented as expected. ${ }^{16-18}$ Finally, the solutions that were proposed provide a rich and original contribution to the literature that would be well heeded by supply and demand stakeholders. They include solutions on how to address the importance of diagnostics in strengthening health systems, where innovation is needed in the production and financing for diagnostics, expanding the evidence base, guidelines 
and evaluation processes, strengthening the supply chain and the quality and capacity of human resources for health.

The participants seemed to regard the technology as the easier part in these dynamics and often discussed technology as if it was separate from the health system. Those who emphasise health system strengthening do so mainly with the aim to improve delivery of diagnostic technologies, not so much based on a comprehensive assessment of technical and social determinants of illnesses and care processes. Thus despite the system thinking there is still a 'silver bullet' aspiration. One participant explicitly warned:

Often a high tech solution is seen as all that is needed, to address what is usually a very poorly understood and defined problem, with complex cultural and systemic issues. I am all for these solutions, if they can bypass these issues, but they are often based on faulty understandings of clinician, patient and healthcare system behaviour. (\#217).

Dropping out of diagnostic and treatment pathways, for instance, does not necessarily happen only because a test result arrived late. What is needed are thorough assessments of the actual problem diagnostic tests are trying to solve and a more honest assessment of the downsides (cost, complexity, maintenance, local and department support) (\#217).

Finding innovative ways to engage and connect different actors involved with diagnostic development and use is paramount for improving development and delivery of new diagnostic technologies. The ongoing aspiration is to investigate how different approaches, such as online discussions, can identify focus areas, contribute to meeting the needs of the decision-making stakeholders and end-users and thereby generate demand for global health diagnostics. While the participants of the online discussions are by no means representative of all stakeholders involved-users, civil society and patients were not sufficiently represented-the online platform allowed for a discussion between globally acknowledged experts in diagnostics and physicians working at the front line and the proposed solutions add to the literature. The lack of involvement of users, civil society and patients likely contributed to the notable absence of discussion around demand creation, access, awareness/education/information and the role of civil society groups and patient representatives in innovation processes. Also, while there were a handful of participants from industry, future discussions could more systematically involve industry representatives to deepen discussions related to diagnostic markets. A useful next step will be to organise a follow-up discussion that allows deepening the discussion in these selected areas. Future discussion panels could further focus on how to gain traction for diagnostic technologies/platforms, business models, interoperability standards, as well as other enabling regulatory or governance mechanisms. Other available frameworks and mechanisms that could be leveraged to further apply the discussion findings: the creation of an essential diagnostics list (similar to the Essential Medicines List by WHO) could address problems with supply chain, import duties, and cost; $;^{19}$ the inclusion of diagnostics into the Global Drug Facility could further improve access to diagnostics; strengthened global diagnostic capacities were emphasised by the commissions evaluating the responses to Ebola ${ }^{20}$ and, finally, diagnostics should be a key element of universal healthcare and primary care strengthening.

Handling editor Soumitra Bhuyan.

Acknowledgements The authors are grateful to the entire GHDOnline community for their active participation in the Expert Panel. They are especially grateful to the 12 Expert Panel members for their time, insights and support: Catharina Boehme, FIND; Jane Carter, AMREF Kenya; Nick Day, Mahidol Oxford Tropical Medicine Unit Thailand; Anthony Emeribe, Medical Laboratory Science Council of Nigeria; Nora Engel, Maastricht University; Dana Goldman, University of Southern California; Jeannette Guarner, Emory University Hospital Midtown; Ilesh V Jani, Ministry of Health Mozambique; Marcel Van Kasteel, Philips; Tsehaynesh Messele, African Society for Laboratory Medicine; Madhukar Pai, McGill University; Rosanna Peeling, London School of Hygiene and Tropical Medicine. They would also like to acknowledge Marie Connelly, Aaron VanDerlip, Aaron Beals, and Marie Teichman for their work in organising and supporting the Expert Panel.

Contributors NE, KW, RW and MP analysed the data. NE, KW, MP, JG, IC $\mathrm{RW}$ and $\mathrm{CB}$ wrote the paper.

Competing interests MP reports that he serves as a consultant for the Bill and Melinda Gates Foundation. NE reports that she serves as a consultant for FIND. CB reports that she is employed by FIND. JG reports that he is employed by the Bill and Melinda Gates Foundation.

Provenance and peer review Not commissioned; externally peer reviewed.

Data sharing statement No additional data are available.

Open Access This is an Open Access article distributed in accordance with the Creative Commons Attribution Non Commercial (CC BY-NC 4.0) license, which permits others to distribute, remix, adapt, build upon this work non-commercially, and license their derivative works on different terms, provided the original work is properly cited and the use is non-commercial. See: http://creativecommons.org/licenses/by-nc/4.0/

\section{REFERENCES}

1. World Health Organization. Global Tuberculosis Report 2015. Geneva: World Health Organization, 2015.

2. UNAIDS. The Gap Report. Geneva: UNAIDS, 2014.

3. World Health Organization. T3. Test. Treat. Track. brochure. Geneva: World Health Organization, 2012.

4. Hung SY, Lai HM, Chou YC. Knowledge-sharing intention in professional virtual communities: a comparison between posters and lurkers. J Assoc Info Sci Technol 2015;66:2494-510.

5. Wenger E. Communities of practice. New York: Cambridge University Press, 1998.

6. Derda R, Gitaka J, Klapperich CM, et al. Enabling the development and deployment of next generation point-of-care diagnostics. PLOS Negl Trop Dis 2015;9:e0003676.

7. Elo S, Kyngäs $\mathrm{H}$. The qualitative content analysis process. $J A d v$ Nurs 2008;62:107-15.

8. Engel N, Ganesh G, Patil M, et al. Barriers to point-of-care testing in India: results from qualitative research across different settings, users and major diseases. PLoS ONE 2015;10:e0135112.

9. Engel N, Davids M, Blankvoort N, et al. Compounding diagnostic delays: a qualitative study of point-of-care testing in South Africa. Trop Med Int Health 2015;20:493-500.

10. Pai M, Yadav $\mathrm{P}$, Anupindi R. Tuberculosis control needs a complete and patient-centric solution. Lancet Glob Health 2014;2: e189-e90. 
11. UNITAID. Malaria diagnostics technology and market landscape. Geneva: World Health Organization, 2016.

12. Schito M, Peter TF, Cavanaugh S, et al. Opportunities and challenges for cost-efficient implementation of new point-of-care diagnostics for HIV and tuberculosis. J Infect Dis 2012;205(Suppl 2):S169-80.

13. McNerney R. Diagnostics for Developing Countries. Diagnostics (Basel) 2015;5:200-9.

14. Pai NP, Vadnais C, Denkinger $C$, et al. Point-of-care testing for infectious diseases: diversity, complexity, and barriers in low- and middle-income countries. PLoS Med 2012;9:e1001306.

15. Pai M, Palamountain KM. New tuberculosis technologies: challenges for retooling and scale-up. Int J Tuberc Lung Dis 2012;16:1281-90.

16. Theron G, Zijenah L, Chanda D, et al. Feasibility, accuracy, and clinical effect of point-of-care Xpert MTB/RIF testing for tuberculosis in primary-care settings in Africa: a multicentre, randomised, controlled trial. Lancet 2014;383:424-35.

17. Dlamini-Mvelase NR, Werner L, Phili R, et al. Effects of introducing Xpert MTB/RIF test on multi-drug resistant tuberculosis diagnosis in KwaZulu-Natal South Africa. BMC Infect Dis 2014;14:442.

18. Albert $\mathrm{H}$, Nathavitharana RR, Isaacs $\mathrm{C}$, et al. Development, roll-out and impact of Xpert MTB/RIF for tuberculosis: what lessons have we learnt and how can we do better? Eur Respir J 2016;48:516-25.

19. Schroeder LF, Guarner J, Elbireer A et al. Time for a Model List of Essential Diagnostics. N Engl J Med 2016;374:2511-14.

20. Gostin LO, Tomori O, Wibulpolprasert S, et al. Toward a common secure future: four global commissions in the wake of Ebola. PLoS Med 2016;13:e1002042. 